\title{
Le barzonisme : un dispositif identitaire complexe en action dans le Mexique contemporain
}

\author{
par Carlos Rafael ReA Rodriguez ${ }^{*}$
}

"La liberté moderne est l'absence de dette". Jacques T. Godbout ${ }^{1}$

I e barzonisme est un courant de mobilisation sociale d'envergure nationale au Mexique, notamment composé de débiteurs n'étant pas en mesure de rembourser leurs crédits bancaires. Cette vaste mobilisation est née dans un contexte de profonde dégradation des conditions de vie de millions de Mexicains, issus des classes moyennes rurales comme urbaines, suite à la crise économico-financière nationale du début des années 1990 ; elle constitue une réponse aux effets de la crise des paiements des crédits bancaires (la crisis de las carteras vencidas) de 1993, aggravée par l'augmentation des taux d'intérêts suite à la dévaluation de la fin 1994.

La mise en œuvre de la politique néolibérale dans le pays (particulièrement la libéralisation démesurée et incontrôlée des secteurs financier et commercial), l'abandon prolongé des petits et moyens producteurs ruraux par l'État et les erreurs répétées en matière de politique économique - débouchant sur " l'erreur de décembre " - ont été d'importants facteurs de détérioration des conditions matérielles de la classe moyenne, et de destruction de son rôle mythique dans l'imaginaire national.

Brusquement, des millions de producteurs, chefs d'entreprise, commerçants, prestataires de services, consommateurs, se sont trouvés en situation de grave crise économique $^{2}$. Des individus ayant emprunté de l'argent aux banques ou aux usuriers, confiants envers les promesses gouvernementales d'une entrée imminente et triomphale dans un monde développé qui assurerait l'accroissement des revenus et du niveau de vie, ont pris conscience qu'ils avaient été dupés. Ils se sont trouvés dans l'incapacité de rembourser leurs dettes, qui avaient augmenté de façon exponentielle avec la hausse des taux d'intérêt durant la crise de 1994, la recapitalisation de ces intérêts et leur décapitalisation accélérée. Les créanciers ont alors décidé de prendre les biens présentés en garantie ; des milliers de procès judiciaires, puis de saisies mobilières et immobilières et de ventes aux enchères ont eu lieu pour faire payer les dettes. C'est donc dans un climat mêlant frustration, déception, peur, impuissance, résignation mais aussi colère et indignation, qu'El Barzón a vu le jour ; d'abord déclenchée dans le monde rural, la mobilisation sociale s'est très vite étendue à la ville.

* Doctorant en sociologie au Centre d'analyse et d'intervention sociologiques (EHESS) et enseignant-chercheur à l'Université autonome de Nayarit (Mexique).

1. J. T. Godbout, Le don, la dette et l'identité, Paris, La Découverte-MAUSS, 2000.

2. On estime globalement que vers 1995 , le nombre de débiteurs s'élevait à environ 8 millions, mais si l'on étend le calcul aux familles concernées, le nombre atteignait presque 40 millions. Vers 1998, les débiteurs étaient environ 11 millions (H. CARTON, El Barzon, clase media, ciudadanía y democracia, Mexico, IIS-UNAM/Plaza y Valdés, 2001). 
El Barzón surgit en août 1993, de façon quasi simultanée dans les États du Jalisco et du Zacatecas, ainsi que dans le centre-ouest et le nord du pays ; la mobilisation s'étend ensuite rapidement dans tous les États. Elle est initialement composée de producteurs ruraux surendettés (petits, moyens et même gros producteurs ; paysans et ejidatarios) ayant des filiations partisanes diverses - proches du Parti Révolutionnaire Institutionnel (PRI) au Jalisco, du Parti Révolutionnaire Démocrate au Zacatecas. Au fil du temps les deux groupes s'affrontent, provoquant en 1994 une grande division au sein du Barzón. D'un côté le Barzón original, qui continue sous le nom de El Barzón Confederación Nacional, est ancré principalement dans l'État du Jalisco et a une influence limitée à l'ouest du pays ; il se compose à l'origine de gros et moyens producteurs faisant de l'élevage et de l'agriculture. Ce Barzón, sous la direction de Maximiano Barbosa, continue à soutenir la lutte des secteurs ruraux affectés par le surendettement. De l'autre côté se développe une nouvelle organisation de débiteurs, El Barzón Unión Nacional, basée au Zacatecas et au Nuevo León ${ }^{3}$, et dirigée par Juan José Quirino ; elle opte pour l'ouverture vers les villes, ce qui lui permet de capitaliser l'impact de la crise financière de 2004-2005, de grossir ses rangs et d'augmenter très fortement son influence. Ultérieurement, deux autres scissions au sein de El Barzón Unión Nacional donneront naissance à El Barzón Movimiento Jurídico Nacional et à La Alianza Nacional El Barzón. Cette pluralité d'organisations se reconnaissant comme barzonistes, ayant les mêmes revendications fondamentales (obtenir une solution au surendettement et à la décapitalisation des secteurs productifs) et identifiant des adversaires communs (les banques et l'État), permet de parler d'un courant de mobilisation que nous nommerons « barzonisme ".

Après 1994, quand El Barzón Unión - l'organisation qui nous occupera désormais - devient un mouvement urbain, les rangs des débiteurs mobilisés se gonflent de centaines de milliers de petits et moyens industriels, de détenteurs de cartes de crédit, de créditeurs hypothécaires, de commerçants, etc., parmi lesquels de nombreuses femmes - ce qui sera dorénavant l'un des traits distinctifs de la mobilisation. L'incorporation de cette pluralité de groupes sociaux urbains donne lieu à des Barzons de secteur, tels que El Agrobarzón, El Transbarzón, El Tortibarzón et même El Barzón des Stars. On y trouve des individus aux opinions partisanes diverses, mais se mobilisant ensemble suite à la décision de El Barzón Unión Nacional de maintenir une position autonome vis-à-vis des organisations politiques. Cette autonomie implique l'alliance avec tout parti disposé à défendre le programme des débiteurs mobilisés (sauf avec le PRI, parti au gouvernement), tout en donnant pleine et entière liberté aux membres de voter pour et de militer dans le parti de leur choix.

Le barzonisme a fait appel à des formes de mobilisation et d'action diverses et créatives : défense in situ des meubles et immeubles présentés en garantie des crédits et menacés de saisie, dénonciation dans les rues et aux portes des bâtiments des gouvernements des municipalités, des États et de la fédération, ainsi qu'à l'intérieur ou l'extérieur des banques et de la bourse mexicaine; actes de résistance dans les bureaux de la justice pour empêcher les ventes aux enchères, lutte juridique auprès des tribunaux; enfin, lutte politico-électorale pour gagner des voix dans les chambres de députés locales et au Congrès de l'Union. Il faut aussi souligner la mise en place de projets de production et de commercialisation alternatifs, ainsi que la négociation

3. Au Zacatecas, il s'agit surtout d'ejidatarios et de paysans pauvres, tandis qu'au Nuevo León (l'un des États les plus urbanisés de la République) il s'agit presque exclusivement de débiteurs citadins. 
avec les différents représentants de l'État et les directions locales et nationales des banques pour trouver une solution globale au problème du surendettement.

On aurait donc ici affaire à une action collective strictement revendicative, n'impliquant pas de réflexion sur le thème de l'identité puisqu'il s'agirait simplement d'un rassemblement d'individus visant la défense de leurs intérêts particuliers. Il n'y aurait ni identité de classe moyenne préexistante à défendre, compte tenu de la situation éclatée de ce segment de la société mexicaine, ni articulation entre les identités particulières des secteurs impliqués. La crise a en effet touché des groupes très hétérogènes, voire aux intérêts contradictoires - les citadins et les paysans, les producteurs et les consommateurs, les agriculteurs et les ejidatarios. Le seul point commun entre ces univers divergents serait la condition juridico-financière de débiteurs insolvables.

À rebours de ces objections, je pense au contraire qu'il est non seulement possible, mais encore indispensable d'analyser le barzonisme dans une perspective identitaire. Je parlerai dès lors ici du barzonisme, et non du barzón au singulier, puisque l'on compte plusieurs barzones nationaux et plus encore de barzones régionaux et locaux ; et si El Barzón Unión Nacional est incontestablement le fer de lance de la mobilisation, les autres organisations restent importantes dans l'analyse du phénomène identitaire. Ce travail se fonde sur la littérature spécialisée sur le sujet et sur des entretiens approfondis ${ }^{4}$, réalisés en août 2000 auprès de cadres dirigeants des deux Barzón au niveau national et de cadres moyens dans les États du Jalisco, du Zacatecas (appartenant aux deux Barzón également) et du Nuevo León (appartenant à El Barzón Unión Nacional).

\section{Remarques préliminaires}

Toute action collective implique au moins trois dimensions fondamentales : la stratégie, la justification et l'identité ${ }^{5}$. Pour agir dans le monde social, en répondant à une situation problématique ou en proposant des initiatives motu proprio, il est indispensable de mobiliser des ressources et des technologies selon des stratégies diverses, et adaptables selon le but à atteindre. Lorsqu'il s'agit de lutter pour des biens collectifs ou publics, justifier les actes est nécessaire pour assurer leur acceptation et leur soutien. À chaque action collective, l'identité fonctionne comme un arrière-plan à défendre, à instrumentaliser, à recomposer ou réinventer, même de manière restreinte et provisoire.

Or dans la société contemporaine émerge, à côté des actions collectives traditionnelles, une myriade de nouvelles expressions de mobilisation sociale résultant des transformations profondes de la société, de plus en plus complexe, de plus en plus difficile à appréhender et de plus en plus délicate à construire. Comme l'affirme François Dubet ${ }^{6}$, il n'y a plus de société pleinement intégrée, de même qu'il n'y a plus de théorie qui en rende compte à partir d'un principe central d'articulation ; par conséquent, il n'y aurait plus de conflits centraux ni de mouvements sociaux capables de jouer le rôle de protagonistes de la grande histoire. Quelles conséquences doit-on en tirer pour repenser l'identité dans l'action collective?

4. Voir la liste des entretiens à la fin du présent article.

5. C. REA, «L'action collective plurielle : la mise en œuvre des grammaires sociales », Paris, polycopié, 2000.

6. F. Dubet, Sociologie de l'expérience, Paris, Le Seuil, 1994. 
Reprenant la thèse de Norbert Elias sur l'identité, selon laquelle il n'existe pas d'identité du Je sans identité du Nous, Claude Dubar ${ }^{7}$ développe l'idée d'une crise majeure dans nombre de sociétés contemporaines, avec pour effet la détraditionnalisation et, en conséquence, la modification du type de relation entre le Nous et le Je, passant de la prédominance du Nous (culturelle et statutaire ${ }^{8}$ ) à la prédominance du Je (réflexif et narratif ${ }^{9}$ ). Les processus de constitution identitaire contemporains répondraient ainsi moins à des attachements communautaires traditionnels qu’à des démarches narratives permettant de gérer les appartenances individuelles à plusieurs collectifs. Souscrire pleinement à cette hypothèse interdirait d'expliquer les nombreuses résurgences communautaires, sauf si celles-ci sont assumées comme des résistances conservatrices face à un destin inéluctable ; elle peut toutefois offrir des pistes de réflexion intéressantes pour les configurations identitaires floues, mobiles, changeantes, flexibles - parmi lesquelles le barzonisme.

Je partirai donc de l'idée que le barzonisme au Mexique est l'une de ces nouvelles formes d'action collective se caractérisant par une très forte pluralité interne, une flexibilité, une nature floue, et un enjeu articulateur dans le registre financier. Je proposerai de dépasser l'analyse de cette expérience sociale en termes strictement instrumentaux pour tenter une autre lecture, en considérant cette expérience comme un dispositif transitionnel de reconfiguration identitaire vis-à-vis des communautés de référence (même si ce n'est pas son but explicite), tout en reconnaissant aussi en son sein l'existence de processus identitaires qui lui sont propres en tant qu'action collective. Enfin, je proposerai d'aborder le barzonisme comme une expression des mutations identitaires du Mexique contemporain, révélant la transformation de la relation Je-Nous via, entre autres, la montée en puissance du discours citoyen et l'épuisement de l'ordre corporatiste d'État.

\section{Les effets de la crise économique}

Au début des années 1990, la crise économique atteint un point critique dans les campagnes mexicaines. Suite au long processus de décapitalisation, accéléré par la dérégulation économique généralisée que prônait le programme néolibéral adopté par le gouvernement fédéral, les producteurs ruraux souffrent de nouvelles pénuries : démantèlement des institutions censées soutenir les paysans orientés vers l'autoconsommation et le marché intérieur (telles la CONASUPO ${ }^{10}$ et la BANRURAL ${ }^{11}$ ), disparition des prix minimum garantis, modification du régime de propriété agraire

7. C. Dubar, La crise des identités, Paris, PUF, 2000.

8. Pour Claude Dubar, la forme identitaire culturelle désigne une configuration où « les individus sont désignés pour leur place dans la lignée des générations et par leur position sexuée dans les structures de parenté " (C. Dubar, ibid., p. 20), tandis que la forme statutaire réfère à une configuration dépendant " de l'apprentissage d'un nouveau code symbolique, de l'intériorisation de nouvelles manières de dire, de faire et de penser, valorisées par le Pouvoir "(C. Dubar, ibid., p. 25).

9. C. Dubar pose que la forme identitaire réflexive consiste « à rechercher, argumenter, discuter, proposer des définitions de soi-même fondées sur l'introspection et la recherche d'un idéal moral » (C. Dubar, ibid., p. 33). C'est pourquoi « elle n'émerge que pour autant que se manifeste une 'distance aux rôles', une temporalité spécifique, celle de l'intimité, du secret ». La forme narrative, pour sa part, «implique le primat de l'action dans le monde et non de la réflexion intérieure sur soi-même [...]. Elle s'organise autour d'un plan de vie, d'une vocation qui s'incarne dans les projets, professionnels et autres. Elle possède comme enjeu l'unité narrative d'une vie, et non la cohérence réflexive d'une intimité »(C. Dubar, ibid., p. 36).

10. Commission nationale d'approvisionnement pour la subsistance populaire.

11. Banque nationale de crédit rural. 
afin de stimuler la concentration pour créer de nouveaux pôles d'accumulation du capital ${ }^{12}$, ouverture incontrôlée des frontières commerciales avec les États-Unis dès avant la mise en place de l'ALENA, etc. S'ajoutent des phénomènes climatiques aux effets ravageurs dans plusieurs régions. Tous ces facteurs brisent la capacité de production, et donc de paiement, de centaines de milliers d'agriculteurs, qui doivent alors solliciter de nouveaux crédits pour subsister et payer leurs dettes. Ce mécanisme enclenche une spirale incontrôlable d'endettement. Les privatisations bancaires et la dérégulation du secteur financier engendrent parallèlement un rétrécissement et un renchérissement du crédit rural ${ }^{13}$; les institutions bancaires deviennent davantage attirées par les investissements spéculatifs que productifs.

Cette crise économique, fortement aggravée en 1993, n'affecte pas seulement les conditions de possibilité de la reproduction matérielle des couches moyennes. Elle ébranle aussi leur position dans l'imaginaire national, ces couches sociales n'étant plus ni la preuve du succès du modèle de développement national-populaire, ni l'image de la réussite possible pour toutes les couches de la population, ni le socle, dans le discours officiel, du sentiment commun d'appartenance à la nation ${ }^{14}$. En peu de temps, elles sont entraînées dans une chute qui bouleverse leur statut et leurs attentes sociales, les arguments historiques légitimant leur propre perception et leur présentation vis-à-vis de l'extérieur, et la reconnaissance dont elles étaient l'objet. La terre, l'école, la petite et moyenne entreprise et la maison, autrefois symboles de leur condition sociale, sont mises en péril.

La crise ne se présente cependant pas partout simultanément. Bien qu'il y ait beaucoup plus de débiteurs en milieu urbain, c'est dans les campagnes du nord (particulièrement à Sonora, Chihuahua et Zacatecas) et de l'ouest (notamment à Jalisco) que l'explosion sociale commence. Cette contradiction n'est qu'apparente et s'explique par le fait que la crise frappe d'abord le milieu rural : dans le modèle de développement de type néolibéral en vigueur dans le pays, notamment depuis la présidence de Carlos Salinas de Gortari (1988-1994), l'autonomie alimentaire est devenue une question obsolète, ce qui marginalise les producteurs ruraux ne pouvant s'insérer dans la dynamique agricole-industrielle d'exportation. La libéralisation des terres agricoles constitue en revanche une priorité nouvelle, pour composer de nouveaux pôles de concentration de capital et faire face aux exigences de la mondialisation

12. Ce qui a eu lieu en 1992, avec la modification de l'article 27 de la Constitution.

13. Parallèlement à la libéralisation mondiale du système financier, le gouvernement mexicain a appliqué dès la fin des années 1980 une réforme bancaire et financière consistant en une disparition de l'encaissement légal à la banque centrale pour affronter des contingences, l'abandon des procédures d'assignation des crédits aux activités prioritaires de l'économie, l'ouverture incontrôlée du système bancaire et financier aux capitaux privés et étrangers, l'élimination de la supervision bancaire. La conséquence de ce virage a été l'instauration d'un système bancaire et financier non solidaire, reposant sur le court terme et à caractère spéculatif, qui a assuré le pouvoir du capital financier. Parmi les données significatives à ce sujet, on note la chute de $80 \%$ du crédit et l'élévation des taux d'intérêt de 15 à $160 \%$ pendant la période 1994-2000. En outre, le régime saliniste (1988-1994) a vendu, entre 1991 et 1992, les institutions bancaires auparavant nationalisées (en 1982), recevant grâce à cette opération 12000 millions de dollars. Après la crise financière de 1994-1995, selon la CEPAL, le sauvetage gouvernemental des banques en faillite s'est monté à 102, voire $117 \mathrm{M} \$$. L'impact du scénario décrit, bien sûr accompagné des conséquences de la mise en œuvre de l'ALENA, est incontestable : à titre d'exemple, en 199510000 entreprises ont disparu, 1 million de Mexicains ont perdu leur emploi, 10 millions de personnes se sont retrouvées en-deçà du seuil de pauvreté (A. RAmíreZ, " México : el desastre bancario ", communication présentée lors du forum Transforming the Global Financial System, Montréal, 2000).

14. Le cinéma et la musique en étant des véhicules privilégiés. 
commerciale. Ainsi, la privatisation et la réduction du crédit, l'abandon graduel de la production rurale en termes de subventions et de prix minimum garantis, et la privatisation virtuelle de nombreuses terres ejidales grâce aux modifications de l'article 27 de la Constitution, sont autant des mesures orientées vers la recomposition radicale de la population rurale et de ses activités productives. Les millions de petits et moyens producteurs touchés (et, au tout début, de gros entrepreneurs), leurs familles et leurs employés deviennent - et sont encore aujourd'hui - les victimes de cette démarche politico-économique.

\section{La crise du corporatisme}

Outre ces phénomènes structurels, la mobilisation sociale puise également à des causes politiques et culturelles. Je rejoins ici Hubert Carton, pour qui la société mexicaine du $\mathrm{Xx}^{\mathrm{e}}$ siècle voit la persistance de formes corporatistes d'action sociale transcendant l'individu, dont la famille élargie est le noyau fondamental et dont les communautés agricoles, l'ejido et les associations de producteurs, sont les principales expressions dans les campagnes ${ }^{15}$. Ces formes ont été les courroies de transmission sociopolitiques privilégiées entre l'État et la société rurale, au sein d'un modèle culturel inspiré des idées d'unité nationale et d'inclusion sociale des masses populaires.

Or cette configuration entre en crise profonde. Face aux processus de transition politique et d'instauration du modèle économique néolibéral, le corporatisme d'État ${ }^{16}$ cède la place à des formes plus contingentes et concurrentielles, mais toujours corporatistes ${ }^{17}$. Dans ce contexte de recomposition sociopolitique, de multiplication des revendications des producteurs en raison de la décapitalisation croissante et de climat de revendication démocratique dans plusieurs États du pays ${ }^{18}$, des réponses organisées deviennent possibles. La tradition associative de la population, les réseaux sociopolitiques existant au niveau régional, le relâchement circonstanciel des contrôles corporatistes et le profond sentiment d'exclusion soudaine du projet national de développement - en tant que secteur productif et d'intérêt, non en tant que citoyens - provoquent l'amalgame des conditions requises pour le déclenchement de l'action barzoniste.

La crise de représentativité au sein des organismes officiels de producteurs à la campagne, qui avaient historiquement maintenu un rapport corporatiste et clientéliste auprès de l'État, ouvre en particulier de nouvelles possibilités d'émergence d'une action collective comme celle d'El Barzón ${ }^{19}$. La crise du corporatisme a lieu

15. H. Carton, El Barzón..., op. cit.

16. Ce qui a été le cas de la centrale ouvrière Confederación de Trabajadores de México, de la centrale paysanne Confederación Nacional Campesina et, pour les éleveurs, de la Confederación Nacional.

17. À l'arrivée des gouvernements néolibéraux au pouvoir, au Mexique, les présidents qui se sont succédé ont frappé fortement quelques-uns des bastions du corporatisme traditionnel, comme les syndicats de travailleurs pétroliers et d'enseignants, entre autres, en remplaçant les dirigeants - qui jouissaient auparavant d'un pouvoir énorme et incontrôlé - par des personnages fidèles au nouveau projet de société. En même temps, à mesure que s'affaiblissait le vieux pacte corporatiste, ont vu le jour de puissants organismes syndicaux qui se sont placés hors de l'orbite officielle, telle l'Unión Nacional de Trabajadores.

18. Ce réveil démocratique a connu un nouvel élan pendant la décennie 1980 : au nord du pays, grâce à la lutte électorale du PAN et au travail de masse des groupes maoïstes ; au centre, à partir des mobilisations sociales qui ont suivi le tremblement de terre de 1985 et les luttes estudiantines de 1986. Au sud du pays, le soulèvement des indigènes connaissait encore une phase de préparation dans l'ombre et le silence.

19. Cf. F. Mestries, "El Barzón o la radicalización de los medianos y grandes productores agrícolas", Sociológica, mai-août 1995, et H. CarTon, El Barzón..., op. cit. 
dans le cadre général du déclin du modèle national-populaire (suite à l'adoption du programme néolibéral en 1982) et des processus de démocratisation politique dans le pays (accéléré après les élections présidentielles de 1988). Ce déclin des organismes corporatistes s'accompagne d'une reconfiguration de réseaux indépendants d'action sociale et politique. Tous ces facteurs catalysent autour de la définition d'un enjeu inédit à la campagne (comme d'ailleurs dans les villes) : le surendettement et l'insolvabilité des agents économiques moyens, phénomène compris comme l'espace d'expression de nouvelles inégalités et d'une nouvelle forme d'exclusion sociale. Il ne s'agit plus d'une inégalité entre ceux qui possèdent des moyens de production et les autres, comme c'était le cas avant, mais entre ceux qui possèdent des ressources directes pour produire des profits à court terme et les autres, même s'ils possèdent leurs moyens de production (par exemple la terre).

Dans les villes en revanche, s'opère un processus plus tardif et individualisé, malgré son caractère massif. Les débiteurs insolvables sont, outre des industriels, surtout des détenteurs de cartes de crédit, des petits commerçants, des créditeurs fonciers ou des acheteurs de voitures, soit une population dont les dettes n'ont que peu ou rien à voir avec les efforts de modernisation visant l'intérêt national. Le relatif retard de la mobilisation urbaine s'explique par ce profil des débiteurs, le recul de la tradition d'organisation politique de la classe moyenne urbaine (hormis certains fonctionnaires et une partie des étudiants) et par son importante fragmentation sociale.

La condition des débiteurs urbains s'est très fortement dégradée jusqu’à la dévaluation de 1994, suivie d'un glissement dans la parité de la monnaie et d'une hausse démesurée des taux d'intérêt pour retenir les capitaux étrangers. El Barzón Unión Nacional $^{20}$, organisation créée en octobre 1994 après la scission du barzón original et fondamentalement orientée vers la mobilisation urbaine, a su se saisir de ce moment. Lensemble du barzonisme, et El Barzón Unión Nacional en particulier, a alors constitué pour de nombreux débiteurs urbains un dispositif privilégié de résonance émotionnelle et d'articulation de leurs revendications, leur fournissant des grammaires permettant de transformer leur situation insoutenable en situation injuste, intolérable et éventuellement transformable.

\section{La blessure identitaire}

On peut ici distinguer plusieurs types de blessures identitaires - c'est-à-dire des états de dégradation, voire de décomposition, des identités sociales préexistantes - des débiteurs. Ces blessures sont éprouvées différemment dans les campagnes et les villes; elles different également selon les États et les régions, les secteurs économiques ou socioprofessionnels, voire selon le type de trajectoire individuelle et familiale. Les ressorts de production de l'indignation sont en conséquence tout aussi variables. En outre, les attentes non manifestes au moment d'adhérer au Barzón et le type concret de dispositif d'action rencontré engendrent aussi une diversité dans les itinéraires de participation.

20. El Barzón Unión Nacional s'est enrichi de milliers de nouveaux membres et sa composition a diamétralement changé, à tel point que, en décembre 1994, environ $80 \%$ de ses membres étaient issus des villes : chefs de petites et moyennes entreprises, commerçants, propriétaires fonciers, possesseurs de cartes bancaires, comédiens, producteurs de pâte pour tortillas, transporteurs, créditeurs hypothécaires, etc. Un autre changement notable a été l'incorporation d'un fort contingent de femmes qui, cette année-là, étaient près de 300000 affiliées (voir G. ToRrEs, "El derecho de barzonear y sus efectos políticos", in J. Alonso et J. M. Ramírez (coord.), La democracia desde abajo, Mexico, Ed. La JornadaCIIH/UNAM-CEEJ, 1997). 
Mais une telle diversité n'est pas infinie, et l'on peut proposer quelques idéauxtypes à partir desquels penser la complexité de la constitution identitaire d'une action collective comme celle-ci. Je voudrais proposer ici trois types généraux de blessure identitaire, se rapportant chacune aux dimensions de la reconnaissance sociale distinguées par Axel Honneth ${ }^{21}$. La perte de "confiance en soi " résulte d'une expérience abrupte ou prolongée de non-reconnaissance des besoins primaires des individus, vécue en termes d'agression ou d'abandon et ayant pour expression émotionnelle la peur. La perte du " respect de soi » est produite par une situation prolongée de non-reconnaissance des droits citoyens, donc de responsabilité morale individuelle vécue comme une expérience d'exclusion et ayant pour manifestation émotionnelle la honte. La perte de "l'estime de soi » enfin est l'effet de situations de non-reconnaissance et de non-valorisation sociale de traits individuels, vécue comme une expérience de discrimination et, émotionnellement, d'humiliation.

Chez les débiteurs ruraux, le type de blessure est globalement d'abord une perte de confiance en soi, situation provoquée par les initiatives judiciaires des banques ayant pour but de leur arracher une large part de leur patrimoine (qui est en même temps source de production et de soutien économique, habitat et objet privilégié du lien intergénérationnel) ; elle devient ensuite perte individuelle et communautaire de l'estime de soi, du fait que ces débiteurs ont souffert de l'exclusion du modèle général de développement. Chez les débiteurs citadins, on trouve surtout une perte de confiance en soi proche de la culpabilité suite à une dépense incontrôlée d'argent ; il y a également perte du respect de soi en raison de la participation croissante, et déçue, à la création de la citoyenneté politique via le vote dans les années précédant la crise.

\section{Stratégies identitaires et motivations latentes}

Pour gérer le décalage entre les identités attribuées et les identités revendiquées, ainsi qu'entre les différentes configurations identitaires au fil du temps, individus et collectifs mettent en oeuvre des stratégies visant à recréer un sentiment d'unité et de continuité de soi.

Parmi les stratégies mises en place par les débiteurs pour faire face aux blessures identitaires subies dans le contexte de crise, on compte le repli (par exemple dans les cas de refoulement lors de pratiques religieuses), l'abandon de soi (cf. les innombrables états dépressifs liés au constat d'impuissance), la haine de soi et la fuite (on pense aux centaines de suicides), la collaboration et l'accommodement (attitudes présentes dans les processus de réaménagement inconditionnel des dettes à l'appel des banques et du gouvernement), la dénonciation et la résistance (réponses liées à l'engagement dans l'action collective) ${ }^{22}$. Albert Hirschman parlerait, lui, de défection, de loyauté et de prise de parole ${ }^{23}$. Les réponses mises en ouvre dépendront de facteurs sociaux multiples et variables, qui révéleront le type de rapport Je-Nous et les dispositifs affectifs, émotionnels, cognitifs, relationnels, organisationnels et institutionnels interagissant dans la situation sociale.

21. A. Honneth propose trois modèles de reconnaissance intersubjective : l'amour, le droit et la solidarité, dont les expressions pratiques correspondantes du rapport individuel à soi-même et aux autres sont la confiance en soi, le respect de soi et l'estime de soi (A. HonNeTH, La lutte pour la reconnaissance, Paris, Cerf, 2000).

22. M. Pollack, Une identité blessée, Paris, Métailié, 1993 et L'expérience concentrationnaire, Paris, Métailié, 2000.

23. A. Hirschman, Défection et prise de parole, Paris, Fayard, 1995. 
Autre élément intervenant dans la réponse des individus, le type de motivation latente lors de l'intégration à la mobilisation. On peut tenter d'en rendre compte a posteriori en distinguant les façons d'évoquer l'organisation, la charge affective et émotionnelle mise en jeu dans les énonciations. Les motivations latentes d'adhésion à El Barzón, mêlées au besoin et au désir de résoudre le problème des dettes, révèlent en effet des recherches personnelles configurant des types de trajectoire en fonction des attentes. On peut ainsi distinguer, dans les entretiens que nous avons réalisés, (1) des recherches ponctuelles d'une solution au problème du paiement (l'engagement dans la mobilisation est alors très faible, voire éphémère) ; (2) des recherches de solidarités primaires, correspondant à des manques affectifs et relationnels dans la trajectoire personnelle, et qui se manifestent dans l'énonciation de l'organisation en termes de famille et de groupe d'amis ; (3) des recherches de solidarité secondaire de type religieux (prédomine ici l'intention réitérée d'aide aux proches); (4) des recherches de réalisation professionnelle et/ou occupationnelle (l'organisation apparaît alors en termes d'école ou de lieu de travail) ; (5) des recherches de nature idéologico-politique (l'invocation de la responsabilité morale des citoyens ou les consignes de la socialisation politique antérieure émergent dans ce cas fréquemment).

Ces trajectoires montrent bien que les motivations de l'action des débiteurs barzonistes ne se limitent pas au registre instrumental mais qu'elles s'étendent aux registres affectif, émotionnel et à celui des valeurs. Les motivations revendiquées par les collectifs ne seront pas une simple addition des motivations individuelles. Cette configuration appelle à des alternatives aux justifications présentes dans les grammaires publiques, ce qui lui confere son intelligibilité et sa force de conviction. Enfin, on note que la tension reste forte entre motivations individuelles et celles du collectif.

\section{La définition intersubjective de l'affront et la montée en généralité}

Comment expliquer le passage de la peur à la réaction, de la honte à l'indignation, de l'humiliation à la colère ? Comment lier les attentes individuelles préexistantes, les circonstances de l'engagement dans l'action et les motifs revendiqués a posteriori de manière collective ? Se référant aux réponses organisées face à la blessure identitaire, Honneth affirme que " de tels sentiments ne peuvent toutefois fournir la base motivationnelle d'une résistance collective que si le sujet est en mesure de les formuler dans un cadre d'interprétation intersubjectif qui les identifie comme typiques d'un groupe tout entier ${ }^{24}$. Laction collective naît donc sous la forme d'un dispositif d'articulation permettant, par une sorte de résonance intersubjective, la coordination des registres émotionnel, cognitif, pratique, argumentatif et identitaire des individus (ce qui n'exclut ni la différence ni le conflit interne). Au principe de cette résonance productrice d'un sentiment de communauté dans le malheur et l'indignation, se trouvent la communication, la reconnaissance des perceptions et évaluations sur le problème commun, et leur reformulation en termes de motifs pertinents et collectivement partagés. C'est dans ce processus qu'apparaissent l'identification et la solidarité.

Le coeur du dispositif des actions collectives conflictuelles est la définition de l'affront dont les individus sont victimes ${ }^{25}$. La définition intersubjective de l'affront

24. A. Honneth, La lutte pour la reconnaissance, op. cit., p. 195.

25. Cf. pour un autre exemple dans le Mexique contemporain S. TAMAYO et X. CruZ, "La marche de la dignité indigène ", Le Mouvement Social, janvier-mars 2003. 
implique de dégager, dans une situation vécue comme problématique, un acte dirigé contre soi - par intention ou par omission -, que l'on stigmatise comme une offense collectivement partagée, insupportable et injuste, et face à laquelle il n'y a d'autre échappatoire que la dénonciation et l'action. La définition de l'affront préfigure ainsi une grammaire permettant de se présenter publiquement comme victime, d'attribuer des responsabilités, de désigner des coupables, des témoins, des arènes de traitement du conflit, des modalités et des procédures de réparation. Elle requiert aussi la définition d'un bien collectif ou public et la revendication de droits défendables, exigibles avec un degré variable de généralité. Il devient ainsi possible de stimuler et canaliser l'émergence de la colère, et de produire des formes spontanées de justice fondant des espaces d'évaluations morales alternatives ${ }^{26}$. Enfin, pouvoir affronter dans un cadre collectif les sentiments de peur, d'angoisse, d'humiliation ou de honte lors de la situation problématique crée les conditions de possibilité d'un renversement du rapport négatif à $\operatorname{soi}^{27}$.

Globalement, l'affront infligé aux barzonistes a d'abord été défini comme l'attaque des banquiers contre le patrimoine individuel et familial ; ensuite, comme une atteinte à la possibilité de reproduction de secteurs entiers de la classe moyenne productive ; enfin, comme une exclusion radicale de secteurs du projet national de développement en application d'une politique économique de type néolibéral. Il y a là un processus de montée en généralité dans lequel se tissent des équivalences avec d'autres secteurs de la population. Il dépasse le caractère collectif de l'affront, du bien concerné et des solutions impliquées, en leur imputant une valeur universelle mettant en jeu non pas un autrui significatif ${ }^{28}$ mais un autrui généralisé ${ }^{29}$, transformant dès lors le champ du conflit, la nature des acteurs et les règles de l'affrontement.

\section{Le dispositif collectif : codification, cadrage et catégorisation}

Il n'existe au départ aucun cadre organisationnel permettant la traduction des situations individuelles des débiteurs en problème collectif, mais des réseaux sociaux et politiques participant à la cristallisation de la résistance et à la définition de leurs premiers codes de signification. On peut a posteriori poser l'hypothèse de la coordination/concurrence entre quatre types de codifications de référence ${ }^{30}$ mises en œuvre lors des débuts de la mobilisation : (1) des codes de type partisan, renvoyant à la propension à participer à la politique institutionnelle chère aux militants ou ex-militants partisans ${ }^{31} ;(2)$ des codes de type corporatiste, liés aux membres ou

26. M. Pollack, L'expérience..., op. cit.

27. Ibid.

28. La notion meadienne d'autrui significatif renvoie aux figures des proches auxquels on s'identifie (C. Dubar, La crise des identités, op. cit., p. 177).

29. Axel Honneth, avec G. Mead, définit l'autrui généralisé comme "le processus de socialisation en général [qui] s'effectue sous la forme d'une intériorisation de normes d'action produites par la généralisation des attentes de tous les membres de la société. En apprenant à généraliser en lui-même les attentes normatives d'un nombre toujours plus grand de partenaires, au point de les ériger en normes sociales d'action, le sujet acquiert la capacité abstraite de participer aux rapports d'interaction de son environnement conformément aux règles qui les réagissent " (A. Honneth, La lutte..., op. cit., p. 95).

30. Il s'agit de manières générales d'interpréter des nouvelles situations sociales selon le précédent milieu de socialisation politique (c'est-à-dire selon le type d'instance organisationnelle) d'où les individus reprennent les clés de lecture primaires pour les affronter.

31. Par exemple, Juan José Quirino, Alfonso Ramírez et Manuel Ortega, connus comme membres du groupe " Zacatecas », provenaient tous du PRD. 
anciens membres de groupes d'intérêts officiels ou indépendants, surtout dans le milieu rural ${ }^{32}$; (3) des codes de type associationniste, en particulier chez les individus (surtout citadins) proches des $\mathrm{ONG}^{33}$ ou de groupes de base, particulièrement en provenance du MUP ; (4) des codes de type individualiste ou citoyen, caractéristiques d'individus n'ayant pas d'antécédents de participation sociale ou politique ${ }^{34}$. La tension entre ces différentes orientations préfigure des dilemmes omniprésents dans le barzonisme : entrer dans la sphère politique ou rester dans la lutte sociale, conserver l'autonomie absolue ou établir des alliances étroites avec un ou des parti(s) politique(s), se concentrer sur des demandes sectorielles limitées ou les diversifier en considérant un univers plurisectoriel, etc.

L'une des opérations symboliques fondamentales permettant de consolider l'organisation des débiteurs est le choix du nom. El Barzón est le titre d'une chanson populaire qui évoque l'exploitation subie par les peones acasillados de la part des hacendados dans le Mexique prérévolutionnaire. Le mécanisme sur lequel se fondait cette exploitation était l'endettement transgénérationnel des peones acasillados dans les tiendas de raya, un des facteurs déclencheurs de la participation de millions de Mexicains à l'éclatement de la Révolution de 1910.

Le choix de ce nom implique donc une opération de cadrage ${ }^{35}$ significative. L'analogie suggérée entre la situation d'exploitation vécue par les peones et celle des actuels débiteurs fait ressortir le caractère injuste, inacceptable, condamnable de l'affront ; s'adressant à l'ensemble des Mexicains, elle dénonce, par une opération de réactualisation de la mémoire historique nationale, l'illégitimité de la conduite des banquiers, mêlant du même coup à la situation un gouvernement supposé préserver les intérêts de la nation.

Avec le temps, un autre cadre vient s'y ajouter. Le discours barzoniste a en effet récemment repris la fonction exercée par l'outil nommé barzón - l'anneau par lequel la barre de la charrue passe dans le joug qui la relie aux animaux ou au tracteur, donnant sa direction à la formation des sillons. Ce nouveau cadrage revendique le rôle unificateur du barzonisme, notamment El Barzón Unión Nacional, à l'égard de l'ensemble de la société civile mexicaine, pour faire face au projet économicopolitique néolibéral.

Outre les types de codes initialement mobilisés et les cadrages déjà évoqués, on peut distinguer dans la trajectoire barzoniste quatre périodes de catégorisation ${ }^{36}$. Les barzonistes se présentent d'abord en débiteurs insolvables refusant de payer, exigeant un moratoire sur leurs dettes et faisant appel aux instances gouvernementales

32. On trouve ici de nombreux secteurs liés auparavant aux unions régionales d'éleveurs.

33. C'est le cas, entre autres, de Liliana Flores Benavides, qui deviendra très vite l'un des principaux leaders de la mobilisation au Nuevo León et au niveau national.

34. C'était le profil de la majorité des débiteurs dans le pays.

35. Pour Daniel Cefaï, «cadrer une situation, c'est pour l'acteur lui reconnaître un air de famille avec d'autres situations déjà rencontrées, et activer des attentes d'arrière-plan qui orientent son attention perceptive, pratique et discursive sur certains thèmes "; tandis que les opérations de cadrage "schématisent des constellations de données en y mettant en saillance des thèmes qui captent l'attention " (D. CefaI, "Les cadrages de l'action collective. Définitions et problèmes ", in D. CefaI et D. Trom (dir.), Les formes de l'action collective. Mobilisations dans les arènes publiques, Paris, Editions de l'EHESS, 2002, p. 3).

36. Selon J. Widmer, « les catégorisations des personnes dépendent de la catégorisation d'une action conjointe, en particulier d'une action énonciative ", les relations internes aux deux catégories étant constitutives d'un dispositif catégoriel commun (J. WIDMER, "Catégorisations, tours de parole et sociologie ", in M. de Fornel, A. Ogien et L. Quéré (dir.), L'ethnométhodologie, Paris, La Découverte, 2001, p. 228). 
pour intervenir en tant qu'arbitres dans le conflit. Ils définissent ainsi une paire catégoriell ${ }^{37}$ en confrontation, des créanciers encaissant des montants injustes par des moyens injustes contre des débiteurs insolvables pour des causes involontaires et ayant dès lors le droit de refuser le paiement.

Ils modifient ensuite leur position en acceptant de rembourser leurs dettes, à condition toutefois de n'en payer que le juste montant. Ils évoluent donc vers la reconnaissance de la co-responsabilité comme issue au problème, ce qui implique le réaménagement des dettes afin d'en éliminer les quantités injustifiées et de leur permettre ensuite de recapitaliser. Les banquiers doivent donc assumer une part du coût, et l'État intervenir pour apporter une solution politique globale et définitive à la crise. La paire catégorielle avec l'État pour arbitre devient donc une triade où tous les acteurs sont directement impliqués et où le témoin invoqué est l'institution judiciaire. Face à la réticence agressive du gouvernement et des banquiers à endosser une part du coût de la crise, le barzonisme entre dans la lutte électorale avec pour objectif d'influer sur les lois.

Se produit alors un déplacement fondamental. Suite à l'application de nombreux programmes de réaménagement des dettes ${ }^{38}$ par le gouvernement et les banques, et face à la démobilisation dans un contexte d'épuisement relatif de l'incursion électorale, les barzonistes formulent une catégorisation nouvelle : ils deviennent des producteurs avant d'être des débiteurs, la nouvelle priorité étant leur recapitalisation et son organisation politique autonome par la filière productive. Les groupes (banquiers), individus (usuriers) ou instances (étatiques) sont alors présentés comme empêchant l'accomplissement du rôle économique des débiteurs, droit historique et citoyen, et privant dès lors l'ensemble de la nation de biens et services indispensables au développement.

Une dernière catégorisation met l'accent sur la dimension de citoyenneté politique, subordonnant désormais celles de consommation et de production. Le barzonisme se trouve de plus en plus confronté aux détenteurs du modèle politique néolibéral au niveau national et mondial, en même temps qu'il lutte pour l'instauration d'une politique économique émanant d'une dynamique décisionnelle démocratique, se fondant sur une notion de marché concurrencé mais solidaire, globalisé mais national.

37. Michel Barthélémy fait référence à la notion de paire relationnelle standardisée comme une relation qui suppose, d'abord, deux catégories dont l'une est le destinateur de l'action et l'autre le destinataire ; et, ensuite, à l'existence entre les deux d'" un foyer de droits et d'obligations qui unit les deux membres de la paire ». Dans cette relation « la seconde catégorie s'approprie les attributs caractéristiques de la première et ceux-ci s'avèrent pertinents en rapport à la situation décrite " (M. BARTHÉLÉMY, «La lecture-en-action : entre le présupposé d'un monde objectif et son accomplissement situé ", Langage et société, septembre 1999, p. 100-101). Dans le même sens, l'auteur propose que des rôles attribués par le rapport entre les catégories, surgissent deux identités opposées dont « l'identité catégorielle du second lui est imposée par l'action dont l'occupant de l'autre catégorie est l'agent » (M. BARTHÉLÉMY, « La lecture ", art. cit., p. 113).

38. Notamment le Fideicomiso de Cartera Vencida (FIRCAVEN), le Crédito a la Palabra du PRONASOL et le Programa Financiero y Técnico del Sector Agropecuario en octobre 1993, le Sistema de Reestructuración de la Cartera Vencida Agropecuaria (Sireca 1 et 2) en mai 1994, les Unidades de Inversion (UDI) en mars 1995, le Cupón Bono Cero, l'Acuerdo de Apoyo Inmediato a Deudores de la Banca (ADE) en août 1995, l'Acuerdo para el Financiamiento del Sector Agropecuario y Pesquero (FINAPE) en juillet 1996, l'Acuerdo de Apoyo Financiero y de Fomento a la Micro, Pequeña y Mediana Empresa (Fopyme) en août 1996, le Programa de Beneficios Adicionales para los Deudores de Crédito para Vivienda en mai 1996, le Programa Punto Final en décembre 1998 (voir H. Carton, El Barzón, clase media, op. cit.). 


\section{Le dispositif transitionnel de reconfiguration identitaire}

L'action collective barzoniste est aussi un dispositif de reconfiguration identitaire du milieu communautaire de départ, c'est-à-dire de reconfiguration du rapport Je-Nous dans les mondes où s'insèrent les individus. Ce dispositif couvre provisoirement leur besoin de reconnaissance et recompose le cadre d'interaction dégradé dans lequel ils ne trouvaient plus leur place.

Claude Dubar affirme, avec Berger et Luckman, que la resocialisation des individus après une crise identitaire requiert " d'abord un dispositif de médiation entre l'ancien soi et le nouveau soi-même. Il faut qu'un partenaire institutionnel, un autrui généralisé, puisse permettre d'accompagner la reconstruction identitaire, de servir d'intermédiaire entre les anciennes identifications en crise, voire en faillite, et les nouvelles en question ${ }^{39}$. Dans la mesure où les divers dispositifs organisationnels barzonistes transforment le problème individuel en problème collectivement partagé, puis en problème de nature publique, ils construisent progressivement un tel autrui généralisé, permettant de dépasser l'état de malheur individuel et ouvrant un espace de réversibilité à l'expérience disloquée.

C. Dubar ajoute qu'est nécessaire « ensuite un appareil de conversation permettant la verbalisation, l'élaboration d'un nouveau langage, mais surtout la rencontre d'un autrui significatif capable de valider, conforter, reconnaître la nouvelle identité latente qui a pu commencer à se dire, qui devient en quelque sorte socialisée " ${ }^{40}$. Recevoir les gens en état de déprime ou d'angoisse dans les premières séances d'information, parler avec eux pour les convaincre qu'il existe des solutions à leurs problèmes, les impliquer dans des actions de résistance (souvent avec succès), constitue un cadre affectif et émotionnel permettant de retrouver confiance en soi et dans le collectif, et de croire à la possibilité d'une solution juste. En fournissant information, formation juridique, économique et politique via des cours, diplômes, assemblées, congrès, etc., un savoir-faire de l'action sociale barzoniste est transmis. À la reconnaissance de soi comme un être responsable et digne, et à la confiance croissante envers les camarades, notamment les leaders, succèdent la solidarité et l'engagement. On fait peu à peu partie d'un nouveau monde commun, d'une communauté restreinte et transitoire de sens où l'on occupe une nouvelle place vis-à-vis des autres.

L'auteur considère indispensable « enfin une structure de plausibilité, c'est-à-dire une sorte de laboratoire de transformation qui permette de gérer la transition entre les anciennes et les nouvelles croyances, les anciens et les nouveaux savoirs, les identifications passées et présentes $"{ }^{41}$. Les processus institutionnels produisant l'identification et la solidarité (des rituels aussi sacrificiels que festifs : les manifestations, les actes de résistance, les occupations des organismes bancaires et gouvernementaux, les cavalcades, etc.) et les espaces de discussion où les membres s'engagent en se mettant à l'épreuve, définissent et cristallisent des identifications et des convictions nouvelles. Leur plausibilité dépend de leur vraisemblance symbolique et de leur efficacité publico-politique.

39. C. Dubar, La crise des identités, op. cit., p. 172.

40. Ibid.

41. Ibid. 


\section{Les processus identitaires dans l'action collective}

Un second niveau dans l'analyse des phénomènes identitaires concernant l'action collective ${ }^{42}$ amène à considérer les processus concrets au travers desquels se produit une communauté de sens se distinguant des autres entités du même système actantiel ${ }^{43}$. En ce sens, et en suivant Donatella Della Porta et Mario Diani, « lorsque nous parlons d'identité, nous ne faisons référence ni à un objet autonome, ni à une propriété des acteurs sociaux, mais plutôt aux processus par lesquels les acteurs sociaux se reconnaissent - et sont reconnus par les autres acteurs - comme faisant partie de groupements plus vastes. Sur la base de telles allégeances, ils signifient leurs propres expériences et leur développement à travers le temps " ${ }^{44}$. On peut distinguer certains axes rendant possible l'identité collective barzoniste : les pratiques rituelles déjà mentionnées, le cadre idéologique prédominant, le programme d'action et les actions politiques concrètes. Les relations personnelles d'inimitié et d'alliance interviennent aussi de manière décisive dans la définition des contours et des relations internes.

L'une des hypothèses permettant d'expliquer la flexibilité politique et identitaire barzoniste est la prédominance des pratiques rituelles sur le discours idéologique ${ }^{45}$ - mais sans les prétentions totalisantes qui caractérisaient auparavant une grande partie des groupes de la gauche sociale et politique au Mexique ${ }^{46}$. Ce sont surtout les actes de résistance, aussi festifs que sacrificiels, tels qu'empêcher quotidiennement les saisies et les ventes aux enchères des propriétés des débiteurs, ou les nombreuses manifestations publiques accompagnées de tracteurs, d'animaux, de musique dans les capitales des États de la République, qui ont stimulé la reconnaissance des débiteurs barzonistes. Au plan idéologique, on distingue un élargissement graduel des demandes barzonistes, permettant de se détacher du seul problème du remboursement des crédits pour se positionner dans le débat sur la définition de la politique économique nationale et, par ce biais, sur la définition du nouvel acteur citoyen capable de répondre aux exigences du temps.

Le courant de mobilisation nationale (donc l'ensemble des barzones) fait aussi coexister des mécanismes flexibles d'identification à divers niveaux, ancrés dans de multiples enclaves (régionales ou nationales, organisationnelles, sectorielles, par strate ou par fonction socioéconomique, etc.). On a donc finalement plutôt affaire à un archipel identitaire ancré territorialement, sur lequel repose un édifice

42. C. Dubar distingue les identités culturelles (héritées) et les identités dans l'action collective (C. DuBAR, "Les identités individuelles et collectives dans le champ professionnel », in M. DE Coster, F. Pichault et A. Touraine (dir.), Traité de sociologie du travail, Bruxelles, De Boeck, 1994).

43. Une dénonciation met en oeuvre « un système de relations entre quatre actants : 1) celui qui dénonce ; 2) celui en faveur de qui la dénonciation est accomplie ; 3) celui au détriment de qui elle s'exerce ; 4) celui auprès de qui elle est opérée " (L. Boltanski, Y. Darré et M. Schiltz, "La dénonciation ", Actes de la recherche en sciences sociales, $\left.\mathrm{n}^{\circ} 51,1984, \mathrm{p} .6\right)$.

44. D. Della Porta et M. Diani, Social Movements. An Introduction, Oxford, Blackwell, 1999, p. 85 (traduction de l'auteur de l'article).

45. C. ReA, "Le barzonisme, un nouveau mouvement social au Mexique? ", Les Cahiers des Amériques Latines, $\mathrm{n}^{\circ} 37$, avril-juin 2001, p. 41-60.

46. Certes les barzonistes luttaient pour une société différente et meilleure, mais ils n'assumaient - à la manière des organisations d'inspiration marxiste - ni route préétablie, ni acteur privilégié, ni destin prédéfini, ni homogénéisation de la société ; ils imaginaient au contraire une société plus juste et démocratique, plurielle et respectueuse de l'initiative des individus, à laquelle il serait possible de parvenir grâce à l'action consciente et organisée des différents secteurs de la population, particulièrement des secteurs productifs. 
organisationnel national produisant de nouvelles identifications plus générales en fonction des exigences pratiques du système d'action conflictuelle dans lequel s'insèrent tous les barzones. Mais l'existence d'un organisme "tête de secteur " au niveau des banquiers, la crise des organismes corporatistes traditionnels de la classe moyenne ${ }^{47}$, la dénonciation dont le gouvernement fédéral fait l'objet en tant que principal responsable de la chute économique des débiteurs et de l'ensemble de la population, ainsi que le rôle des médias traitant de façon indifférenciée les différentes organisations barzonistes, ont permis la consolidation d'un système d'action conflictuelle d'envergure nationale.

On peut alors penser à un processus identitaire majeur englobant des identités particulières multiples sur le plan local-régional. « Une identité qui inclut des identités mineures et qui est flexible ne déterminera pas strictement le critère d'appartenance à un groupe. En d'autres termes, on n'associera le mouvement ni à un groupe social particulier ni à une idéologie spécifique, à un style de vie ou à un code symbolique ${ }^{48}$. Dans ce réseau complexe de champs identitaires multiples, il s'agit bien davantage d'une dynamique polycentrique ${ }^{49}$ que d'une quelconque structure hiérarchique centralisée. On distingue toutefois quatre axes qui commencent à organiser - de façon encore précaire - l'ensemble de la mobilisation : l'enjeu financier général, qui affecte tous les barzonistes ; le recours dans leur discours, avec des effets unificateurs croissants, aux figures du citoyen, du producteur et du consommateur ; le rôle protagoniste du Barzón Unión, qui donne une direction générale à l'ensemble de la mobilisation ; enfin, le traitement indifférencié de l'action des divers barzones par les médias, qui offre, de l'extérieur, un cadre d'identification générale commun. Mais bien qu'il y ait des axes d'articulation identitaire globale, les tensions et les contradictions entre les différentes orientations internes ne disparaissent jamais totalement.

La dynamique identitaire se déroule en plusieurs phases. D'abord, la phase de résistance directe dans les rues et pour la défense des biens en danger, dans laquelle se tissent les toutes premières identifications dans la négativité permettant l'expansion nationale de la mobilisation (1993-1994). Puis une phase de décantation idéologicopolitique, qui se dédouble suite à la première grande scission (1994). Ensuite, les débuts de l'affirmation de la force collective, quand les débiteurs organisés passent à la lutte juridique et lancent des défis importants au système judiciaire lui-même (1995-1997). Suit une phase de subordination à la logique partisane, et de résistance à une telle subordination par certains secteurs du barzonisme, dans un contexte d'incursion dans la lutte électorale en alliance étroite avec le PRD (1997-2000). Puis une phase d'éloignement de la logique partisane et de recentrage sur les processus organisationnels et identitaires de la mobilisation, particulièrement de la part du Barzón Unión Nacional (2000-2002). Enfin, une dernière période s'ouvre lors du sommet de Monterrey en mars $2002^{50}$; le barzonisme, à la tête duquel se trouve le Barzón

47. C'est le cas particulier, au tout début, des Unions régionales d'élevage à la campagne.

48. D. Della Porta et M. Diani, Social Movements, op. cit., p. 105 (traduction de l'auteur de l'article).

49. Voir C. Calhoun, in D. Della Porta et M. Diani, Social Movements, op. cit.

50. À l'occasion de la Conférence "Financement pour le Développement ", convoquée par l'ONU à Monterrey Nuevo León, Mexico, en mars 2002, El Barzón Unión Nacional a appelé les débiteurs de la banque de toute l'Amérique Latine à participer à la Conférence Internationale « Financement : Dette ou Développement ", le 11 et 12 mars 2002, dans la même ville de Monterrey. Parmi les assistants à la conférence, on a compté des débiteurs du Venezuela, du Costa Rica, de la Colombie et du Mexique, ainsi que des épargnants de l'Argentine (voir la page web de El Barzón Unión Nacional, www.elbarzon.org). 
Unión, choisit la voie de l'internationalisation de la mobilisation en rencontrant des débiteurs et des épargnants de plusieurs pays d'Amérique latine. Cette orientation, indispensable pour réanimer la lutte, représente des opportunités d'action très importantes et des défis nouveaux sur le terrain identitaire ; la situation représente toutefois aussi un risque de fuite en avant, révélant l'impossibilité de pouvoir affronter avec succès les défis d'une imminente baisse en généralité de la mobilisation ${ }^{51}$.

Fin 2002 et début 2003, les barzonistes se sont engagés dans une importante lutte paysanne. Tout en demandant la renégociation du chapitre agricole de l'ALENA avec les États-Unis et le Canada, les producteurs mexicains, notamment ceux produisant des cultures vivrières, ont exigé de l'État un soutien pour affronter la concurrence des producteurs étrangers - fortement soutenus par leurs gouvernements - et la garantie de réelles possibilités de survie. Après une mobilisation ayant articulé plusieurs organisations paysannes du pays, des négociations ont eu lieu avec l'État mais, aux yeux des paysans, sans résultats suffisants.

El Barzón a dès lors essuyé une baisse constante de sa capacité d'action, pour cause de démobilisation d'un grand nombre de ses membres suite aux solutions proposées au problème du surendettement. Cette situation a obligé les barzonistes à se replier sur des revendications rurales, les campagnes subissant toujours une décapitalisation de l'économie aggravée par les effets de l'ouverture commerciale avec les États-Unis et le Canada, avec pour conséquence le maintien de fortes tensions sociales et de possibilités d'action collective. Les secteurs ruraux du barzonisme ont en outre toujours montré un niveau plus élevé d'intégration et d'identification à la mobilisation. La catégorisation barzoniste tend donc à s'affaiblir à mesure que s'abaisse son niveau de généralité ${ }^{52}$.

\section{Conclusion}

Le barzonisme est donc un mouvement ni culturel ni identitaire ; mais il est impossible de comprendre sa capacité à perdurer dans le temps de manière articulée sans prendre en compte les dynamiques par lesquelles se constituent son identité ainsi que les effets de son action sur les milieux sociaux dont sont issus les débiteurs.

Il ne s'agit plus de la recherche d'une identité perdue. Si, d'une certaine façon, les actions collectives des classes moyennes contemporaines essaient de reconstituer au Mexique les circuits «traditionnels » de reproduction de leur sociabilité (la terre, l'entreprise, l'école, etc.), il existe un autre horizon de justification, de type postconventionnel, qui émerge en modifiant la nature du processus : on fait référence à l'intégration de valeurs civiques, composantes de plus en plus centrales dans les revendications.

Enfin, l'analyse du barzonisme permet d'émettre une hypothèse plus générale sur l'évolution du rapport Je-Nous dans la société mexicaine actuelle. D'une part,

51. Il est intéressant de noter qu'El Barzón Union n'a pas essayé de s'agréger au mouvement altermondialiste.

52. Aujourd'hui, El Barzón Unión Nacional a décidé de se transformer en une Fondation qui « entend récupérer toutes les expériences, bonnes et mauvaises, de la lutte de El Barzón, dans tout le pays [...], dans le but de systématiser l'information et de créer une bibliothèque nationale de ce mouvement [...]. Nous sommes le seul mouvement social dans le monde qui a vaincu la politique de dépouillement [...]. La Fondation El Barzón organisera six ateliers par an [...] pour apprendre aux simples citoyens la défense des droits humains économiques et sociaux » (site Internet d'El Barzón, www.elbarzon.org). 
face à la sévère décomposition sociale résultant de la crise générale du pays, certaines actions collectives représentent des îlots de configuration d'identités sociales et politiques restreintes, comblant provisoirement les vides de la socialisation existante. D'autre part, le rapport de ces actions collectives au domaine identitaire montre un mélange prometteur entre affirmation croissante des valeurs citoyennes, en faveur de l'émancipation graduelle du Je face au tout pouvoir, et affirmation de dynamiques d'action collective mobilisant de façon créative et moderne des références imaginaires de type communautaire, le zapatisme en étant l'exemple paradigmatique ${ }^{53}$.

On peut y voir des manifestations organisées d'action collective, bien qu'isolées, faisant face à l'épuisement d'un modèle identitaire global subordonnant les individus à des communautés sociopolitiques intermédiaires, elles aussi subordonnées à un pacte corporatiste d'État en décomposition. En termes strictement identitaires, le barzonisme participe ainsi dans les faits, peut-être sans le vouloir et même sans le savoir, aux affrontements dont émaneront de nouvelles actions collectives déjà en germe, ainsi que de nouvelles configurations identitaires, bases des cadres de socialisation et de l'image des individus à venir.

\section{ENTRETIENS RÉALISÉS EN 2000}

- Alfonso Ramírez (président national d'El Barzón Unión Nacional), 18 août.

- Liliana Flores (secrétaire nationale d'El Barzón Unión Nacional), 20 août.

- Manuel Ortega (secrétaire national à l'action politique, El Barzón Unión Nacional), 7 août.

- Apolinar Barbosa, (président d'El Barzón Confederación Nacional en Jalisco), 30 août.

- Miguel Estrada (président d'El Barzón Unión Regional en Jalisco), 30 août.

- Pedro Ortega (président d'El Barzón Unión Nacional à Zacatecas), 25 août.

- Socorro Cabrera (El Barzón Unión Nacional à Nuevo León), 20 août.

- Hilda Hernández (El Barzón Unión Nacional à Nuevo León), 20 août.

- Imelda Hernández (El Barzón Unión Nacional à Nuevo León), 20 août.

- Leonardo Martínez (El Barzón Unión Nacional à Nuevo León), 20 août.

- Esthela Ortega (El Barzón Unión Nacional à Nuevo León), 20 août.

- Simona Reyna (El Barzón Unión Nacional à Nuevo León), 20 août.

53. Voir aussi S. TAmayo et X. Cruz, "La marche... ", art. cit. 